\title{
PROPOSAL FOR A WEB PORTAL MANAGING REGISTRATION FOR STUDENT ACCOMMODATION IN A DORMITORY
}

\author{
Ivor PODUNAVAC, Dominika CRNJAC MILIĆ, Krešimir NENADIĆ
}

\begin{abstract}
A proposal for developing a database model and a web portal for managing registration for student accommodation in a dormitory is given in this paper. The portal has two user profiles and corresponding functionalities; they are intended for users/students and administrators, respectively. In the process of application for student accommodation in a dormitory, every student has to provide data referring to GPA, i.e. academic achievement at school, level of education, university, parents' status, household members, the number of siblings and their school age, and household monthly income. There are also extra conditions that could ensure direct allocation of accommodation at a dormitory. After the application process deadline has expired, the administrator can either accept or decline applications. At the end of the application process, the final ranking list can be published and made accessible. Advantages of using a web portal for the dormitory accommodation election and application process are e.g. better accessibility and the fact that students are exempt from delivering the necessary application documentation personally. In that way, costs are reduced, efficiency at work is increased and the possibility of making errors when entering and processing data is significantly lower
\end{abstract}

Keywords: application; database model; dormitory; student; web portal

\section{INTRODUCTION}

This paper describes the process of development and utilization of a web portal. The developed web portal enables easier application process to potential candidates for student accommodation in a dormitory. Web portal also enables the ranking process, which creates final ranking list of possible dormitory beneficiaries according to the rent price. The ranking is calculated according to high school average grade or passed exams, achieved ECTS (European Credit Transfer and Accumulation System) points, year of studying, social status, etc.

The second section gives a short overview and analysis of current methods of ranking students.

The third section presents technologies used to develop database and web portal.

The fourth section explains development process and related functionalities in web portal.

The fifth section concludes this paper.

This paper is a result of a student graduate thesis written by Ivor Podunavac at Josip Juraj Strossmayer University of Osijek, Faculty of Electrical Engineering, Computer Science and Information Technology in Osijek, Croatia. [1]

\section{SHORT OVERVIEW}

This section of paper will give insight in the manner the applications are handled without support of ICT (Information Communication Technology).

\subsection{Current Methods of Ranking Students against Using New Technologies}

Throughout the month of July students and future students have the option to apply for a student dormitory accommodation. First-year students and older have to deliver their application documents personally, while prospective students can send their application documents by mail. The first step in applying for student dormitory accommodation is to pick up the application form at the dormitory. The next step is to fill in the application form and include all the other required documents. The application form requires the following data to be filled in:

- student name and last name,

- name of university and faculty student is applying to,

- total number of achieved ECTS credits,

- number of ECTS credits achieved in current academic year of studying,

- certificate of passed exams, and

- whether the studying program is in a science filed that is marked as deficit on the national level.

The web portal developed as a part of student thesis enables insight in all the required conditions in one place. Another advantage of the web portal is in finishing the application in significantly less time than using paper forms, and web portal user's interface is friendlier. This paper will give a short overview of the older paper-form version of the application process for the dormitory. Work efficiency as well as data processing and creating final rang list will be also mentioned.

Advantage of using new technologies in applying for accommodation can have different groups of beneficiaries, for example high school pupils and students.

Major difference in using new technologies during application process is much easier accessibility, lower costs, and all the relevant data can be processed much faster regarding the server. Applicants can use the web portal functionality on any device with internet connection (a computer, a smartphone or a tablet) and they can finish the application process in several minutes. The only presumption is that all the required documents have already been prepared on the device that will be used for the application process. 


\subsection{Current State of Accommodation}

There are 14 dormitories for student accommodation in eight cities throughout Croatia. Student dormitory in Slavonski Brod has the smallest number of beds - 115, while student dormitory "Stjepan Radić" in Zagreb has the largest number of beds $-4,014$. Total sum of all available beds in dormitories for student accommodation across the Republic of Croatia is 11,709. Every year the capacity for student accommodation in dormitories across the Republic of Croatia has maximum occupancy. Every year the total number of applications for student accommodation in dormitories scales about $35-40 \%$ above the total available capacity (data acquired by direct inquiry in dormitory in Osijek - similar situation in majority of dormitories). Every summer in Croatia between 15,000 and 17,000 students apply for accommodation in dormitory in a way that they supply all the required paper documents to a certain dormitory they want to apply to. That does not depend on their current residence.

There is a tendency to expand capacity to accommodate more students in dormitories. Two new student dormitories have been opened in the academic year 2017/18. One is in Knin, with a maximum capacity of 78 beds (https://www.veleknin.hr/veleknin/web/index.php/Studentsk $\mathrm{i}$-dom) and the other one is in Virovitica, with a maximum capacity of 108 beds (http://studom.vsmti.hr/o-domu/1/).

There is a web portal of similar type already in use. It is for student accommodation in dormitories at the University of Zagreb [2]. This web portal has restricted access and allows access only for students of the University of Zagreb. Still, majority of dormitories for student accommodation in the Republic of Croatia do not use the type of application process that will be presented later in this paper. Hence, all students applying for student accommodation in dormitory have to enclose all the required paper documents. There is a problem that students from distant places spend more money for travel expenses and they spend more time for the application process. There is a limited number of available beds so it is possible that some students will not get the desired accommodation. The application process starts after all the applicants enclose all the required documents and the closing date expires. Student centre or student dormitory employees register, manage and process all the supplied data in available software solution for the application process. This is the right place to observe efficiency and profitability of current manner of conducting the application process against the way proposed in this paper. Students could perform the process of registration and filling in the data for themselves. Employees would only have to verify the documents supplied through the web portal. Verification process is required to check that all the applicants supplied all the documents to avoid possible rigging (average grade certificate, some kind of disability or incapacity, year of studying, etc.).

Unlike the way students have applied so far, this is more affordable and cost-effective with regard to minimizing student costs associated with applying, time and cost of data processing, as well as greater control of the data entered. In particular, the ease of use of such a web portal can be emphasized. It is sufficient to have access to the internet and that it is possible to complete the entire application for accommodation in the dormitory within several minutes by simple handling.

\section{TECHNOLOGIES USED IN DATABASE MODELLING AND WEB PORTAL DEVELOPMENT}

Technologies used in database modelling and web portal development are:

- Cascading Style Sheets (CSS) for styling the content a user can see,

- HTML (HyperText Markup Language) for setting content,

- PHP (PHP: Hypertext Pre-processor) as a server side scrip language to perform all thr functionalities,

- JavaScript (JS) for manipulating and verifying data on the client side, and

- MySQL (Structured Query Language) for manipulating data in the database.

\subsection{HyperText Markup Language}

HTML is simple to use and easy to learn and these are the main reasons of its worldwide acceptance and popularity. It is intended to be free to use f or any user from its very beginning. Web browsers are applications available to parse and present content from HTML documents [3]. HTML is not a programming language, nor the people using it are programmers. It is simply used to create HTML documents that contain some information for users.

\subsection{Cascading Style Sheets}

CSS is language for styling content in HTML documents that will be presented to the user in a web browser. [4] CSS can be used to have an impact on the view of particular document elements, such as images, links, text, or the whole page and even on arranging element positions. Styles are usually organized in external files separated from HTML document. In this way, the visual identity of more HTML documents can be changed by editing and changing single CSS document. External CSS files allow more flexibility and control in arranging the document's presentation.

\subsection{PHP - Hypertext Pre-processor}

PHP is an open-source server-side script language. It allows HTML code to be dynamically generated. Opensource means that anybody may download PHP source code written in programming language $\mathrm{C}$ and add new functionalities. PHP is one of the most widely used serverside script languages.

Entire PHP source code has to be written between tags $<$ ?php and ? > There are no restrictions on how many times PHP tags can be used in a PHP document. One has to be careful when using PHP tags to correctly use the opening $(<$ ?php) and closing tags (?>). 


\subsection{MySQL}

MySQL is a free to use, open-source database management system. SQL is a structured language used to generate queries into a database. MySQL databases are of relational type because it is proved to be the best way of storing and searching a vast amount of data. SQL database is the base of almost every information system, i.e. the base of every business subject that needs availability and quality of information.

Every MySQL database can have more than one user with available access. Every user can have predefined access rights. In this manner, good database access settings can significantly reduce the possibility of errors. MySQL as a database management system can operate on wide variety of operating systems. [5]

\subsection{Bootstrap Framework}

Bootstrap is a free development framework that can make creating a web portal easier. It consists of templates written in HTML and CSS for different components of graphical user interface, as well as JavaScript extensions. That is why it is called a development framework for web applications.

It is compatible with all the web browsers. Some of advantages using Bootstrap in a web portal development are as follows: easier and faster development, easier implementation of web forms, supported responsive design, and its ease of use. There are some deficiencies, such as visual similarities between web applications developed using Bootstrap, library reloading every time a web page is reloaded which leads to slower page loading.

It is not necessary to use Bootstrap in a web page development, but it can save time if a developer uses it.

\subsection{JavaScript - Client Side Script Language}

JavaScript is the most popular client-side script programming language. It was created with a goal to introduce interactivity to HTML documents. All web browsers support it. Netscape developed JavaScript in 1996. It is interpreting script language, which means that the script code is executed one instruction at a time without compiling whole script code and creating executable file.

JavaScript code is executed on the client side. Code written in JavaScript can be placed in HTML document between tags <script> and </script> inside the document's head element or inside the body tags. JavaScript code can also be written as an outside code in separate .js file and included (called) from any HTML document linked to it. [6]

\section{WEB PORTAL DEVELOPMENT}

This web portal supports two operating modes: user mode and administrator mode. Both are explained further in this section.

\subsection{Database Structure}

Database is created to enable storing and accessing data for the web portal. All the required information is stored in tables in the database. Database used in this web application is called studentskidom (student dormitory) and consists of four tables. Tables rezultati (results) and dokumenti (documents) have a foreign key referring attribute ID in table korisnici (users). Foreign key ensures database integrity and prevents deleting data from other tables where foreign key from this table is the primary key in another table. There can be only one primary key per table. Column in the table tagged as primary cannot be empty and has to be unique for the designated table. Primary key value is automatically auto incremented one by one upon inserting a new row into the table. Column (attribute) idKor in the table rezultati (results) is the foreign key and points out the user who applies for student dormitory accommodation. Similar table organization is for table dokumenti (documents).

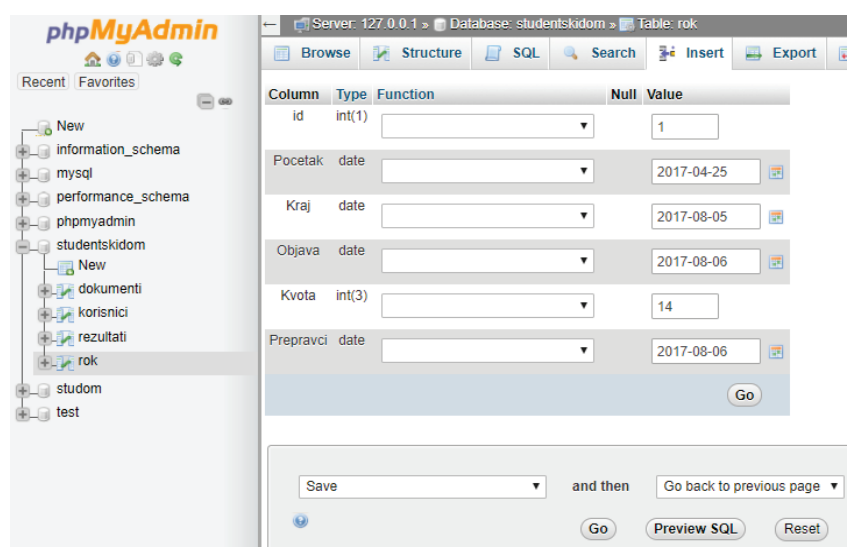

Figure 1 Table rok (deadline) in database modelled in phpmyadmin application

Fig. 1 shows the database table rok (deadline) modelled in free PHP application phpmyadmin. All the tables in database are modelled with the same application because it has a user-friendly interface and it has no requirements for any specific foreknowledge concerning programming.

Table rezultati (results) stores data about students applying for accommodation (year of studying, faculty, score for each category).

Table korisnici (users) contains attributes id, name, encrypted password and unique code given to the user upon registration. Unique code distinguishes users rather than the first and the last name. This mechanism ensures transparency of ranking students who can find and recognize only their result by the given unique code.

Table dokumenti (documents) consists of document's names attached by the student in the application process. Uploaded documents ensure validity of all the relevant data during the application process. Each document is named according to the unique code given to the user by the web portal. 


\subsection{User Registration}

Before using the web portal, it is necessary to finish the registration process. After the user correctly fills in the required data, they become a registered user and can login and use the web portal's functionality. As mentioned earlier, every new user receives a unique $I D$ by calling uniqid() function. This function generates a string of characters based on the current system time. During the registration process the user can select their status - a high school graduate or student because there is a difference whether they apply for the first time as a high school graduate or as an older student.

Every user has to enter password required for login to the web portal. The passwords are encrypted by hashing thus making them unrecognizable to other users, including web administrator as well.

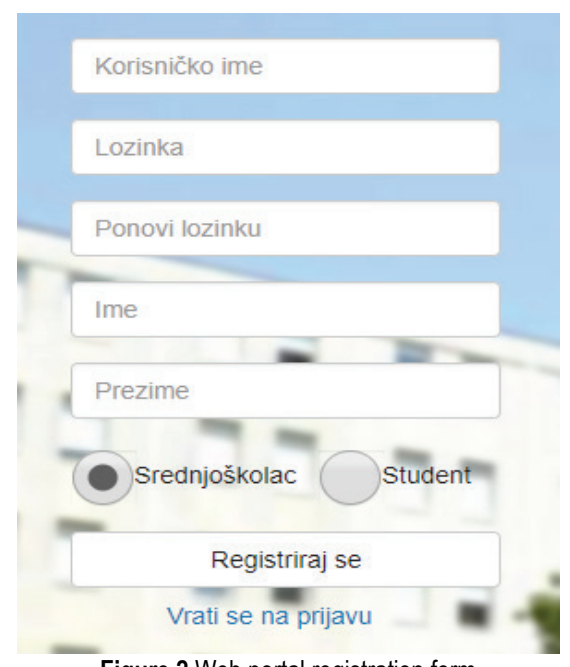

Figure 2 Web portal registration form

Figure 2 shows the registration form included in the web portal. The data required for registering are visible on the register form.

\subsection{Accommodation Application Process}

Portal home page requires user to enter the username and the password. In the case the user enters valid information (the user is registered and password is correct for that user), the portal will redirect the user to the page for filling in the required data during the application process for student accommodation in the dormitory.

During the login process, every user is assigned to their own session, which differs from other sessions by logged username. Existence of session data is checked in each server-side script. When the session data do not exist, the user is redirected to the login page.

\subsection{User Mode Functionality}

The user can access the portal's functionalities after the successful login. There is an option to access personal data and fill in the main form. Here is an option for uploading the required documents. Option for dormitory provides basic information about two dormitories for student accommodation in Osijek. A registered user can find results here if the ranking process is completed.

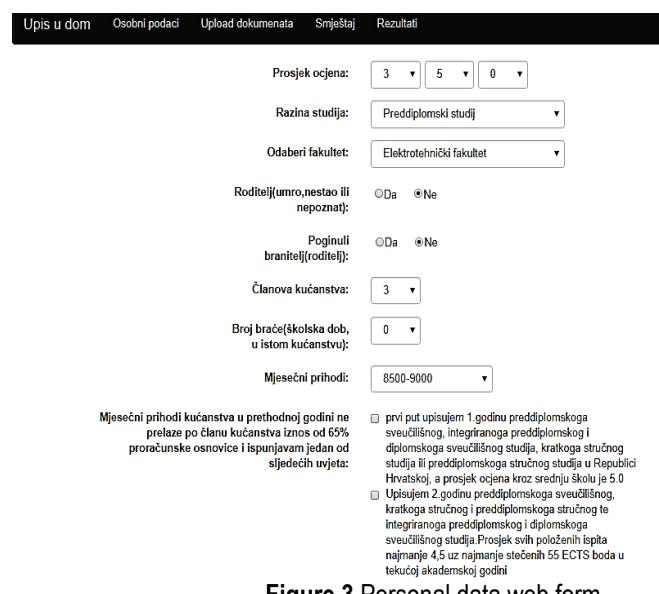

Figure 3 Personal data web form

The web page for personal data filling in process consists of a form that requires filling in the average grade, the level of education (high school graduate or student), the faculty, parents (both parents alive or just for one parent), the number of residents in domicile household, the number of siblings that are pupils or students, and the parents' monthly total income. There are conditions that ensure direct accommodation in the dormitory (a student without both parents, a parent killed in war - both for students with 4.0 or higher average grade, etc.). If the applicant meets some of the above listed conditions, it is required to confirm their status by uploading relevant documents. An applicant can change personal data until the application process deadline expires. The web portal has a functionality that checks all the relevant data by cross-referencing uploaded documents. If the student's application is not valid, there is a short period for making required changes and upload missing documents.

The application form for student accommodation in dormitory has to be filled in as well, alongside the check of all the necessary uploaded documents. The web portal can check ECTS points to ensure the student has the minimum number of ECTS points to apply for student accommodation in a dormitory. The Ministry of Science and Education prescribes the minimum number of required ECTS points.

The required documents have to be in digital form in order to upload them to the server. There can be restrictions to the form of required documents, like PDF (Portable Document Format) or word document (doc or docx).

\subsection{Ranking Results}

The publication of results is expected in several days after the final deadline expires. All applicants can check and enclose any missing documents in the case administrator rejected the application due to incomplete application data or documentation. The whole process of calculating and ranking applicants is completely automated and performed by PHP server-side scripts. 
The applicant can achieve additional 200 points by applying to deficit professions designated by the Ministry of Science and Education.

If the applicant does not have one parent, they can achieve additional 600 points. If they are a child of a defender killed in war, they can achieve additional 200 points.

Each sibling adds additional 150 points. A number of residents in domicile household has to be filled in in the application. If the total income per residence is less than 500 HRK, the applicant achieves the maximum number of 750 points in this part of the application. The number of achieved points is decreased until total house holding income becomes less than $65 \%$ of prescribed budget base. If the total house holding income is greater than 1,900 HRK and less than $65 \%$ of prescribed budget base $(2,162 \mathrm{HRK}$ in the time the web portal was developed), the applicant will achieve the minimum number of 75 points. If the total household income is greater, the applicant will not achieve any points in this category.

Based on the criteria for direct accommodation, each applicant can choose one of the listed conditions, corroborate selected condition by uploading relevant documents related to it, and thus achieve direct accommodation in dormitory regardless the number of achieved points.

There is a PHP server-side script that calculates achieved points based on the applicant's average grade. After this calculation is completed, all points from different categories are added to the total number of achieved points. Ranking list is calculated after the application deadline expires and all the applicant's points are calculated.

There is a PHP function (prosSum ()) that calculates points for all faculties and for all applicants that are at the same year of studying.

All applications have to be validated by the administrator by checking all the uploaded documents and crossreferencing them with appropriate categories in each applicant's form.

There is a small difference between high school graduate applicants and applicants that are already students. The difference is in the way the points are calculated.

There are two attributes in the table rezultati (results) Pregledan (inspected) and Valid. Their default values are set to ' 0 ' (zero). After the administrator examines applicant's data and documents, the decision to reject or accept application is made. If the application is rejected, the applicant has several days to complete the application and upload missing documents and fill in the required data.

Figure 4 shows the table for some test application data. The majority of applied users were successful to apply for student accommodation in dormitory, but there are two unsuccessful applications. There were 14 vacancies, which means that 14 applicants will achieve accommodation in the dormitory. Applicants that have direct student accommodation in the dormitory are at the top of the ranking list followed by the rest of applicants ranked by the achieved points. As can be sseen, there are no names on the ranking list but unique IDs provided by the web portal.

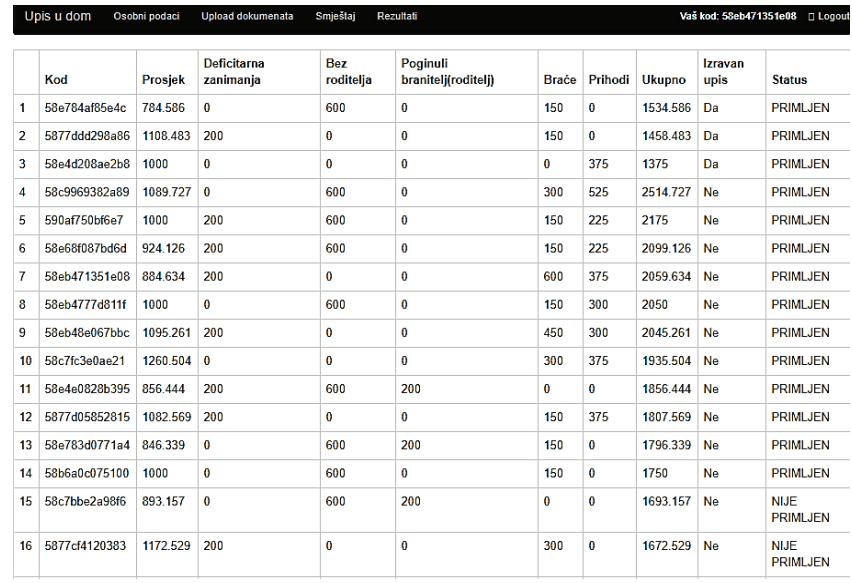

Figure 4 Test data rank list

\subsection{Administrator Mode Functionalities}

An administrator (or administrators) is (are) logged to the web portal as any other user. Authorisation is performed by checking the entered username and password against the data in the users table in database. Fig. 5 shows starting the web page after the administrator is successfully logged in.

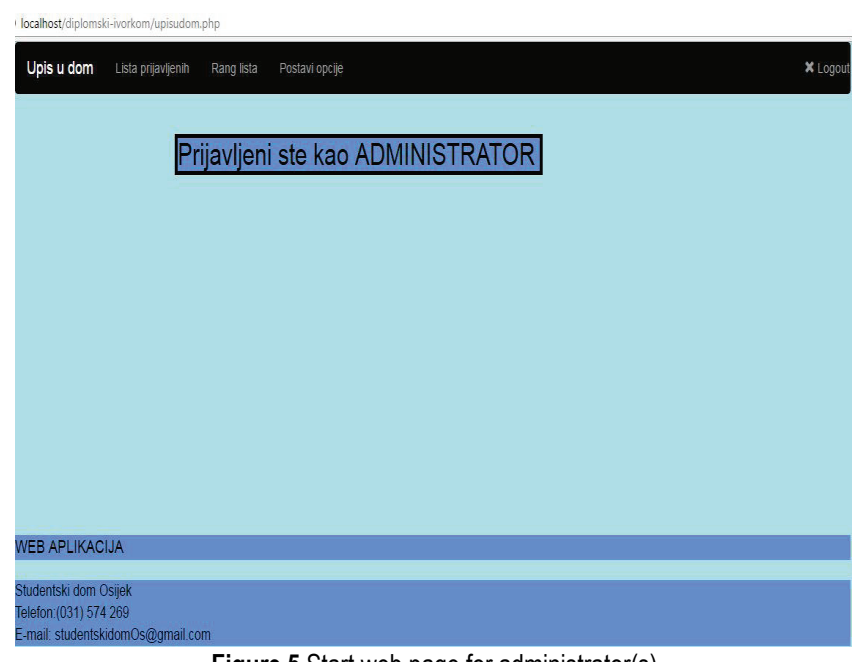

Figure 5 Start web page for administrator(s)

The administrator can set options for starting and closing date, incomplete correction deadline date, publishing ranking list date and number of vacancies in dormitory according to the deadline published in the newspapers.

After the applicant has fulfilled all their relevant data and uploaded all the required documents, it remains to wait for expiration deadline date if the application was successful and complete.

\subsubsection{Registered Applicants' Checklist}

A registered applicants' checklist can be observed as a waiting list. The administrator can see applications rejected due to incomplete documentation or missing data. If the applicant completes their application by uploading additional required documents or filling in the missing data, administrator can confirm the application afterwards. The 
opposite result would be rejecting the application because of incomplete documentation or missing data.

If the administrator rejects the application, a popup window will show requiring the administrator to enter additional explanation. This explanation will be shown to the applicant the next time they $\log$ in the web portal. The final deadline to complete the application will be shown as well.

Fig. 6 shows the list of applicants the way an administrator can see it. There are lists of documents organized in columns (as shown in Fig. 6) and the administrator can open attached documents (Otvori), accept (Prihvati) or reject (Odbij) the application.

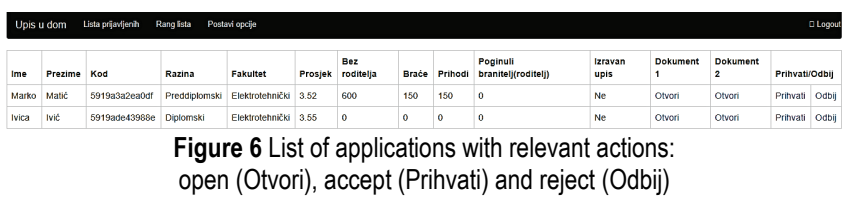

A ranking list is created and shown in the Rang lista (Ranking list) option in the menu after the deadline date expires. All applications accepted by the administrator will be shown in the ranking list.

\section{CONCLUSION}

The developed web portal offers simplicity and a userfriendly environment because it can be accessed by using any available web browser on any device connected to the Internet. The web portal was developed by using modern web technologies like CSS, HTML, PHP, JavaScript, and MySQL was used for database management. This web portal is ready to be used for dormitories in Osijek and with minor changes, it can be used for any student or any high school dormitory.

There are several advantages of using the developed web portal, e.g. applicants are not required to personally deliver their application forms and the required documents; a smaller number of Student Centre employees is required to check out the applications; the process of creating a ranking list is automated and it is performed almost instantly, to name a few.

There is a possibility of communication between applicants and web application administrators by exchanging messages. This enables applicants to be informed of any change in the application process.

The only requirement this web portal imposes is Internet connection. This can be considered as a drawback, but there is a greater number of advantages. Unlike today's application to high school and student dormitories, this approach is more available and cost-efficient. It minimizes the data processing time and the cost of human resources engaged in manually filling in the required data from documentation. It also reduces student costs (e.g. transport) and the possibility of entering incorrect data in the web form.

\section{REFERENCES}

[1] Podunavac, I. (2017). Model i programska podrška za rangiranje studenata pri upisu u studentski dom (in Croatian), graduate thesis, Josip Juraj Strossmayer University of Osijek, Faculty of Electrical Engineering, Computer Science and Information Technology in Osijek.

https://zir.nsk.hr/islandora/object/etfos\%3A1400

[2] See https://natjecaj.sczg.hr/student

[3] See https://www.mojwebdizajn.net/skriptni-jezici/vodic/html/ uvod-u-html.aspx

[4] See https://www.w3.org/Style/CSS/Overview.en.html

[5] See http://php.com.hr/77

[6] See http://razno.sveznadar.info/10-doc-PDF/2_MySQL.pdf

[7] Haverbeke, M. (2011). Eloquent JavaScript: A Modern Introduction to Programming, William Pollock, San Francisco.

\section{Authors' contacts:}

Ivor PODUNAVAC

Josip Juraj Strossmayer University of Osijek

Faculty of Electrical Engineering, Computer Science and Information Technology in Osijek

Kneza Trpimira 2B, 31000 Osijek, Croatia

ivor.podunavac@etfos.hr

Dominika CRNJAC MILIĆ, Full Professor

Josip Juraj Strossmayer University of Osijek

Faculty of Electrical Engineering, Computer Science and Information Technology in Osijek

Kneza Trpimira 2B, 31000 Osijek, Croatia

+38531224681, dominika.crnjac@ferit.hr

Krešimir NENADIĆ, Associate Professor

Josip Juraj Strossmayer University of Osijek

Faculty of Electrical Engineering, Computer Science and Information Technology in Osijek

Kneza Trpimira 2B, 31000 Osijek, Croatia

+385 31495 429, kresimir.nenadic@ferit.hr 